\title{
CT Diagnosis of Foreign Body in Urinary Bladder after Surgical Management of Stress Urinary Incontinence
}

\author{
Han-Jui Lee, Tzu-Pin Lin, Shu-Huei Shen, Jia-Hwia Wang \\ Department of Radiology (HJL, SHS, JHW), Division of Urology and Department of Surgery (TPL), \\ Taipei Veterans General Hospital and National Yang-Ming University School of Medicine (HJL, TPL, \\ SHS, JHW), Taipei, Taiwan
}

This 49-year-old Taiwanese woman presented with voiding urethral pain, frequency and hematuria. She had the past history of diabetes mellitus, hypertension and schizophrenia. The labor history was G7P2. She had suffered from stress urinary incontinence (SUI) for several years and received repair surgery using polypropylene mesh sling (SPARC ${ }^{\text {TM }}$ sling system) two years ago. Since one week after the surgery, she had had several episodes of urinary tract infection and no specific cause could be identified. This time she was admitted for the same symptoms. The most recent urianalysis showed positive for occult blood, white blood cell (WBC) 11-20/ high power field and many bacteria. The urinary culture yielded Escherichia coli more than $10^{6}$ colonies/ mL.

KUB showed an amorphous hyperdense material in left lower pelvis (Figure-1). Computed tomography (CT) study revealed a $3 \mathrm{~cm}$ curvilinear hyperdense lesion at left anterolateral wall of urinary bladder (Figure-2). Under the impression of intravesical foreign body, cystoscopy was performed which revealed the misplacement mesh with stone encrustation (Figure-3). It was removed by Thulium Laser. There was no pyuria in the follow up urianalysis (WBC 0-2/ high power field) and her symptoms were improved.

Mesh repair has been used for surgical treatment of SUI since 1990s and has gained popularity. Urinary tract injury is a known complication of the procedure, which may be due to iatrogenic injury during the surgery or subsequent erosion of the synthetic mesh through the mucosa (1). The incidence of urinary tract injuries from mesh repair is not exactly known, with higher reported rate in synthetic material (2-4). The definite diagnosis of urinary bladder foreign body is made by cystoscopy, and endoscopic or surgical removal of the foreign body effectively cure the symptom (1). However the diagnosis is usually delayed with the median of 24 months after the original surgery, because the presenting symptoms are variable and confused with recurrent SUI or post-sling obstruction symptoms (1-3,5-7). This is the first report of the imaging findings of urinary tract injury following SUI surgery. In patient with previous history of pelvic surgery, calcified urinary tract foreign body on CT should alert the present of iatrogenic injury.

Figure 1 - The plain KUB at postoperative 29 months showed a small irregular round radiopaque material (black arrow) at left lower pelvis which was not seen on the postoperative 1 week film (not shown).

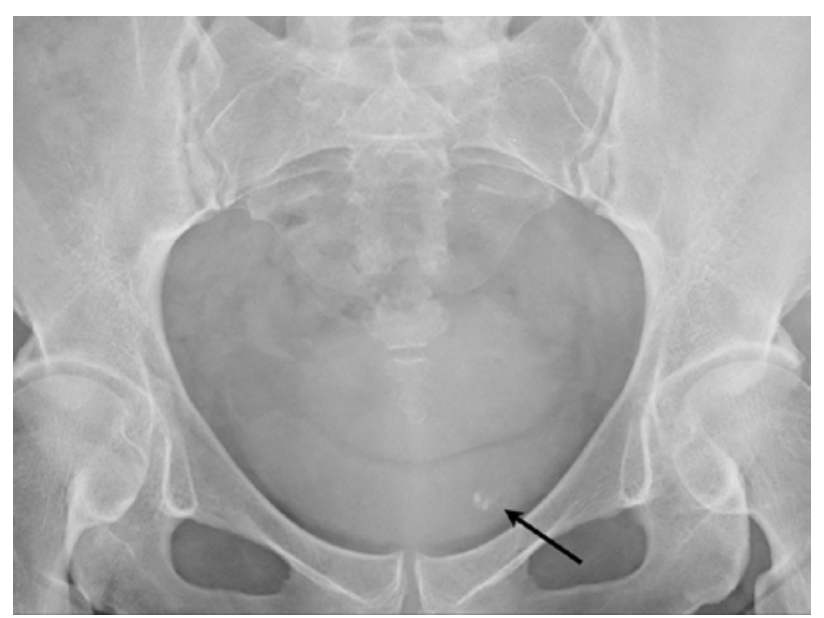


Figure 2 - Axial scan of pelvic CT, (a) noncontrast and (b) contrast enhanced, bone window. A curvilinear hyperdense material (black arrow) was demonstrated at left anterolateral wall of urinary bladder.
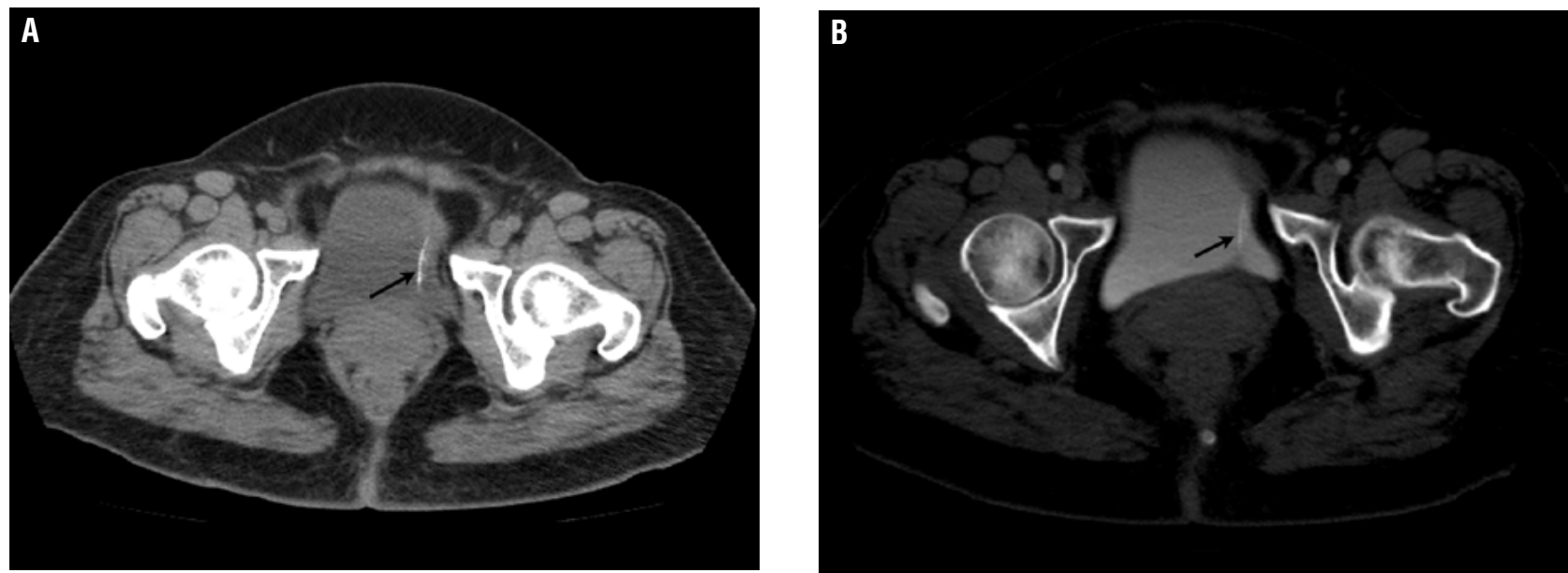

Figure 3 - Cystoscopy demonstrated misplaced suture (long arrow) with stone encrustation (short arrows).

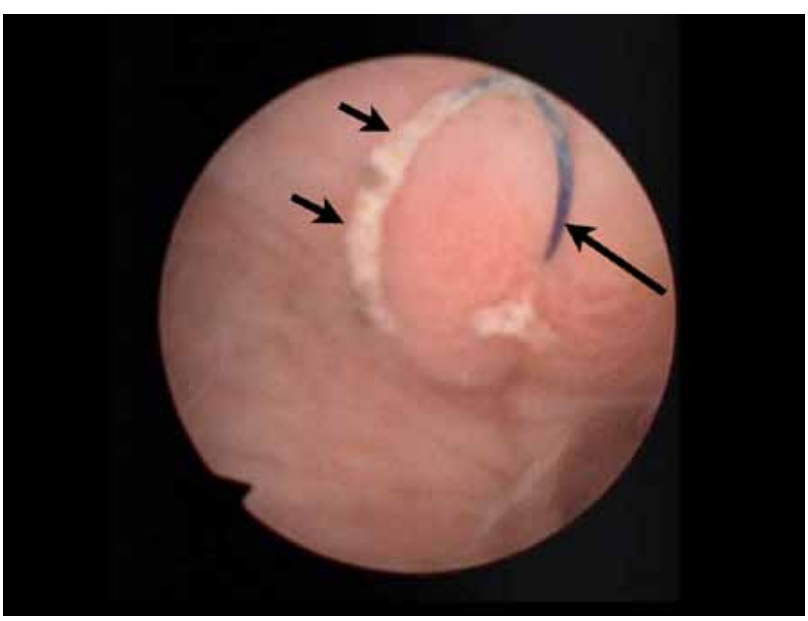

\section{REFERENCES}

1. Frenkl TL, Rackley RR, Vasavada SP, Goldman HB: Management of iatrogenic foreign bodies of the bladder and urethra following pelvic floor surgery. Neurourol Urodyn. 2008; 27: 491-5.

2. Dmochowski RR, Blaivas JM, Gormley EA, Juma S, Karram MM, Lightner DJ, et al.: Update of AUA guideline on the surgical management of female stress urinary incontinence. $J$ Urol. 2010; 183: 1906-14.

3. Clemens JQ, DeLancey JO, Faerber GJ, Westney OL, Mcguire EJ: Urinary tract erosions after synthetic pubovaginal slings: diagnosis and management strategy. Urology. 2000; 56: 589-94.

4. Atherton MJ, Daborn JP, Tsokos N, Jeffery JT, Yin MJ: Complications associated with tissue anchor migration after vaginal surgery using the tissue fixation system - a case series. Aust N Z J Obstet Gynaecol. 2012; 52: 83-6.
5. Baessler K: Do we need meshes in pelvic floor reconstruction? World J Urol. 2012; 30: 479-86.

6. Deval B, Haab F: Management of the complications of the synthetic slings. Curr Opin Urol. 2006; 16: 240-3.

7. Tse V, Chan L: Outlet obstruction after sling surgery. BJU Int. 2011; 108(Suppl 2): 24-8.

\section{Correspondence address:}

Dr. Shu-Huei Shen Department of Radiology, Taipei Veterans General Hospital, Taiwan School of Medicine, National Yang-Ming University, Taiwan 201, Sec. 2, Shipai Rd., Taipei 112, Taiwan

Fax: + 8862 2876-9310 E-mail: shshen@vghtpe.gov.tw 